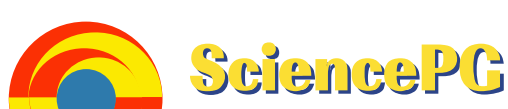

\section{Anthrax: A Re-Emerging Livestock Disease}

\author{
S. Parthiban ", S. Malmarugan, M. S. Murugan, J. Johnson Rajeswar, P. Pothiappan \\ Department of Veterinary Microbiology, Veterinary College and Research Institute, Tamilnadu Veterinary and Animal Sciences University, \\ Tirunelveli, Tamilnadu, India
}

Email address:

parthis17@gmail.com (S. Parthiban)

\section{To cite this article:}

S. Parthiban, S. Malmarugan, M. S. Murugan, J. Johnson Rajeswar, P. Pothiappan. Anthrax: A Re-Emerging Livestock Disease. International Journal of Nutrition and Food Sciences. Special Issue: Review on Novel Approaches for the Management of Emerging and Reemerging Livestock Diseases. Vol. 4, No. 4-1, 2015, pp. 7-12. doi: 10.11648/j.ijnfs.s.2015040401.12

\begin{abstract}
Anthrax is a contagious and highly fatal zoonotic bacterial disease affecting primarily herbivores. Mortality can be very high, especially in herbivores. The disease has world-wide distribution and is a zoonosis. The etiological agent is the endospore-forming, Gram-positive, nonmotile, rod-shaped Bacillus anthracis. Central to the persistence of anthrax in an area is the ability of $B$. anthracis to form long-lasting, highly resistant spores. Understanding the ecology of anthrax spores is essential if one hopes to control epidemics. Studies on the ecology of anthrax spores have found a correlation between the disease and specific soil factors, such as alkaline $\mathrm{pH}$, high moisture, and high organic content. The repeated anthrax outbreak in livestock and subsequent infection to human has been considered as a nationwide alarming issue. Outbreaks of anthrax have diverse consequences on society. Establishing the appropriate control strategies is very important and crucial in reducing the socio-economic impact of the disease. Control measures are aimed at breaking the cycle of infection, and their implementation must be adhered to rigorously. It can be used as a biological weapon and has been classified as a Category 'A' bio threat by Centers for Disease Control and Prevention(CDC). This review describes this important disease covering its etiology, epidemiology, transmission, pathogenesis, clinical signs, diagnosis and prevention and control strategies to be adopted to combat this globally important pathogen.
\end{abstract}

Keywords: Anthrax, Ecological factors, Epidemics, Management strategies

\section{Introduction}

Anthrax was the first disease for which a causative bacterium, Bacillus anthracis, was positively identified. The disease derives its name from the Greek word for coal, anthrakis, due to the coal-like black lesions found on the skin in cutaneous anthrax [1]. Robert Koch developed a method for culturing pure $B$. anthracis in 1876 . Anthrax is a naturally occurring disease with worldwide distribution caused by the bacterium Bacillus anthracis. This bacterium exists in nature in two forms as an active growing vegetative form or as a dormant spore. The spores are very hardy and tolerant to extremes of temperature, humidity, and ultraviolet light. They can survive for long periods of time (even decades) in the environment without nutrients or water because of its hardy spores, B. anthracis can survive for extended periods in the environment, a trait that likely contributed to the successful global spread of this organism [2]. Control of anthrax is one of the top priorities to reduce economic losses and to protect the valuable animals susceptible to this disease because the spore forming bacteria that can remain alive but dormant in the soil for many years. The bacteria can "bloom" and contaminate surface soil and grass after periods of wet cool weather, followed by several weeks of hot dry conditions. To have a better preventive and control measures, the factors influencing the occurrence of anthrax should be identified. The present study is undertaken to know the influence of soil $\mathrm{pH}$, soil organic carbon, population density, migrating flock, common grassing land, predators and scavenging carriers on the occurrence of anthrax in the endemic and non-endemic areas. Anthrax occurs in animals such as cattle, sheep, goats, deer and horses they ingest anthrax spores when they consume contaminated grass and water.

\section{History}

Anthrax is intertwined with the origins of modern microbiology and immunology. Anthrax was the first disease 
in history proven to be caused by a microbe. French bacteriologist Casimir-Joseph Davaine first observed the presence of anthrax bacilli in infected sheep's blood in 1863 . In 1876, Robert Koch, through his research in Wollstein, Germany, went on to empirically prove that the anthrax bacillus was the etiological agent of anthrax. He did so by isolating the bacilli from cutaneous lesions of sheep, obtaining pure cultures by growing the bacilli on the aqueous humor of ox's eye, and proceeding to inject the bacteria into uninfected sheep [2]. In 1881, Louis Pasteur successfully produced a vaccine to protect livestock against anthrax, the first effective live bacterial vaccine to be developed.

\section{Epidemiology of anthrax}

\subsection{Biology of Bacillus Anthracis}

B. anthracis is an aerobic, gram-positive, non-motile rod. The bacterium measures $1-1.5 \mathrm{~mm}$ by $3-10 \mathrm{~mm}$. Spore formation occurs centrally or paracentrally and causes no bacterial. Spore formation occurs when nutrients are depleted as happens after host death and exposure to air [3]. B. anthracis spores are highly resistant to various environmental changes and can survive indefinitely in soil, air, water and vegetation despite extreme heat or cold, dessication, chemical treatment or ultraviolet exposure. The highly resistant nature of the spore aids in the persistence of the disease in an area [2]. The bacteria grow readily on all conventional microbiology media at $37 \mathrm{C}$ including sheep blood agar and produce non-hemolyticcolonies. Colony appearance on agar is typically $4-5 \mathrm{~mm}$ rough, white colonies with a characteristic comma shape or tail often referred to as "curly-hair" or "medusa head" colonies [4]. B. anthracis occurs singly or in pairs in tissue and forms long chains in culture giving a classic "boxcar" appearance [3]. B. anthracis is part of the B. cereus group of bacilli which includes B. cereus, B. thuringiensis, and B. mycoides Anthrax can be differentiated from other members of the group by several methods. All members of the B. cereus group, except B. anthracis, are resistant to penicillin because of a chromosomally encoded betalactamase. Other characteristics, which differentiate B. anthracis from other Bacillus species, are the absence of hemolysis, lack of motility and the presence of an antiphagocytic capsule consisting of D-glutamic acid [4]. It is theorized that spore germination may be triggered inside the macrophage by host-specific signals such as elevated temperature $\left(>37^{\circ} \mathrm{C}\right)$ and $\mathrm{CO}_{2}$ concentrations $(>5 \%)$ and presence of serum components [2].

\subsection{Transmission}

Outbreaks of anthrax tend to occur in association with particular climatic and weather events, such as heavy rainfall, flooding and drought. In anthrax-prone areas, the close grazing of animals on fresh shoots of grass after rainfall often leads to outbreaks of the disease due to the ingestion of organisms picked from contaminated soils. During severe outbreaks, biting flies may transmit the disease from one animal to another but this is a very minor mode of transmission. The principal mode of transmission is ingestion of infective micro-organisms. Non-biting blowflies may contaminate vegetation by depositing vomit droplets after feeding on a carcass infected with B. anthracis. Animals feeding on such vegetation then become infected. Wild carnivores become infected through the consumption of infected animals that have died from anthrax. Outbreaks of anthrax have been reported in some animals (mainly pigs) after ingestion of feeds containing meat and bone meal based concentrates originating from carcasses contaminated with anthrax bacterial spores [3].

\subsection{Factors Associated with Anthrax Epidemics}

The moisture content of soil mainly depends on its type, which may influence in the long time persistency of anthrax spore. Loamy type soil increases anthrax outbreak, soil with moisture range between $6.31-28.37 \%$ might be favorable for the viability of $B$. anthracis spore in the soil. The soil $\mathrm{pH}$ controls the availability of many nutrients in soil. Alkaline soil containing high nitrogen, calcium $(\mathrm{Ca})$, and organic matter gives favorable condition to the spore for growing in soil. The soil that is rich in organic matter and calcium promotes the survival of resilient $B$. anthracis. In addition to adequate $\mathrm{Ca}$, nitrogen and organic matter in the soil, anthrax outbreak requires favorable seasonal changes such as warm weather followed by heavy rain. The bacteria are thought to undergo a vegetative cycle when the above conditions are fulfilled. By this process, anthrax spores could be concentrated in top soil to cause disease in grazing animals, occurring outbreak separated by disease-free intervals [5].

In livestock's anthrax epidemics also occurs from the anthrax non endemic regions and causes huge economic loses. The main precipitating factors contributing anthrax in non-endemic area includes, heavy flood which will wash out the anthrax spores from the soil and enrich the non endemic area and by the scavengers and carnivores which may feed on the dead carcass which are died due to anthrax and spreads the anthrax spores in the non endemic areas through its feces and excretions.

\section{Virulence Factors}

Bacillus anthracis clearly owes its pathogenicity to two major determinants of virulence: the formation of a poly-D-glutamyl capsule, which mediates the invasive stage of the infection, and the production of the multicomponent anthrax toxin which mediates the toxigenic stage.

\subsection{Poly-D-Glutamyl Capsule}

Bacillus anthracis forms a single antigenic type of capsule consisting of a poly-D-glutamate polypeptide. All virulent strains of $B$. anthracis form this capsule. Production of capsular material is associated with the formation of a characteristic mucoid or "smooth" colony type. "Smooth" (S) to "rough" (R) colonial variants occur, which is correlated 
with ability to produce the capsule. $\mathrm{R}$ variants are relatively avirulent. Capsule production depends on a 60 megadalton plasmid, pX02. The poly-D-glutamyl capsule is itself nontoxic, but functions to protect the organism against complement and the bactericidal components of serum and phagocytes, and against phagocytic engulfment and destruction. The capsule plays its most important role during the establishment of the infection, and a less significant role in the terminal phases of the disease, which are mediated by the anthrax toxin. The poly-D-glutamyl capsule is formed in vivo or in the laboratory when the bacterium is grown on serum plates in a $5 \% \mathrm{CO}_{2}$ atmosphere. The capsular material can be detected by the McFadyean reaction which involves staining with polychrome methylene blue. Blue rods in a background of purple/pink-stained capsular material are a positive test. Neither $B$. cereus nor $B$. thuringiensis synthesizes this capsular polymer, so the detection of capsular material can be used to distinguish $B$. anthracis from its closest relatives [6].

\subsection{Anthrax Toxin}

The toxigenic properties of Bacillus anthracis were not recognized until 1954. Prior to that time, because of the tremendous number of anthrax bacilli observed in the blood of animals dying of the disease $\left(10^{9}\right.$ bacteria $\left./ \mathrm{ml}\right)$, it was assumed that death was due to blockage of the capillaries, popularly known as the "log-jam" theory. But experimentally it was shown that only about $3 \times 10^{6}$ cells $/ \mathrm{ml}$ is necessary to cause death of the animal. Furthermore, the cell-free plasma of animals dying of anthrax infection contained a toxin which causes symptoms of anthrax when injected into normal guinea pigs. These observations left little doubt that a diffusible exotoxin plays a major role in the pathogenesis of anthrax [2].

One component of the anthrax toxin has a lethal mode of the action that is not entirely understood at this time. Death is apparently due to oxygen depletion, secondary shock, increased vascular permeability, respiratory failure and cardiac failure. Death from anthrax in humans or animals frequently occurs suddenly and unexpectedly. The level of the lethal toxin in the circulation increases rapidly quite late in the disease, and it closely parallels the concentration of organisms in the blood. The bacilli secrete three proteins, protective antigen (PA), lethal factor (LF), and edema factor (EF), and these proteins combine to form the lethal (PA plus LF) and edema (PA plus EF) toxins. PA is the common cell binding component and is required for toxin activity. Two large virulence plasmids, $\mathrm{pXO} 1$ and $\mathrm{pXO} 2$, encode toxin production and the formation of a poly- $\gamma$-d-glutamic acid capsule respectively [6].

\section{Pathogenesis}

The disease manifests itself in one of three forms: cutaneous, inhalational or gastrointestinal depending upon the route of spore entry [7]. The two latter forms, inhalational and gastrointestinal anthrax, are the most fatal and rare. Cutaneous anthrax accounts for up to $95 \%$ of all anthrax infections throughout the world and is mainly due to occupational exposure. The most common areas of exposure with cutaneous anthrax are the head, neck and limbs. Spores are often introduced subcutaneously via a cut or skin abrasion, although skin trauma may not be required. Incubation periods after spore exposure generally range from 1-10 days. Initial symptoms often present as a painless, pruritic papule that resembles an insect bite at the site of infection. The papule becomes vesiculated in 1-2 days with occasional hemorrhage. These vesicles rupture to form depressed ulcers with focal edema that develop the characteristic dry necrotic black center. Generally the disease will remain localized, however, patients may develop systemic symptoms including fever, malaise and headache. Antibiotic treatment does not halt the progression of the papule to ulceration. Differential diagnosis includes brown recluse spider bite, cellulitis, ulceroglandular tularemia, accidental vaccinia, ecthyma gangrenosum, and cat scratch disease. Gram stain and culture of any lesions are recommended for diagnosis before antibiotic treatment is initiated [2]. The mortality rate for cutaneous anthrax without antibiotic treatment is reported as $20 \%$, while with antibiotic treatment, death is rare. Inhalational anthrax occurs as the result of spore deposition in the alveolar spaces of the lung. Historically, inhalational anthrax is a rare occupational disease of people who worked with raw wool, hence the name "wool-sorters disease" [3].

Cattle and sheep can die quickly from anthrax, but their carcasses may show no obvious signs of the disease. But the length of the illness varies and some animals may have signs of illness for several days before death. In such cases the main clinical signs are high temperature, shivering or twitching, harsh dry coat, blood in dung or in nostrils, decrease or complete loss of milk, fits, bright staring eyes, colicky pains and dejection and loss of appetite. In pigs and horses anthrax can cause death in pigs and horses, though less quickly than in cattle and sheep. The main clinical signs of anthrax in pigs and horses are hot painful swellings in the throat area, sudden colic pain in horses and loss of appetite in pigs. Rigor mortis or stiffening is not complete. Necropsy reveals haemorrhages in the internal organs and the spleen is almost always enlarged.

\section{Diagnosis}

Diagnosis of anthrax based on clinical signs alone is difficult. Confirmatory laboratory examination should be attempted if anthrax is suspected. Because the vegetative cell is not robust and will not survive 3 days in transit, the optimal sample is a cotton swab dipped in the blood and allowed to dry. This results in sporulation and the death of other bacteria and contaminants. Pigs with localized disease are rarely bacteremic, so a small piece of affected lymphatic tissue that has been collected aseptically should be submitted. Before submission, the receiving reference laboratory should be contacted regarding appropriate specimen labelling, 
handling, and shipping procedures. Specific diagnostic tests include bacterial culture, PCR tests, and fluorescent antibody stains to demonstrate the agent in blood films or tissues. Western blot and ELISA tests for antibody detection are available in some reference laboratories.

\subsection{Direct Examination of Smears}

Anthrax is diagnosed by examining Romanowsky stains applied blood or other body fluid samples, or tissue specimens collected within a few hours of death from animals for the presence of the bacteria. Samples must be collected carefully to avoid contamination of the environment and to prevent human exposure to the bacteria. Blood samples from relatively fresh carcasses will contain large numbers of B. anthracis, which can be seen under a microscope. Fixed blood smears stained with Loeffler's Polychrome methylene blue (MacFadean reaction) stains can be used and the capsule visualized; however, this can result in some $20 \%$ false positives [3].

\subsection{Bacterial Isolation}

All Bacillus species grow well on 5\% sheep blood agar and other routine culture media. Polymyxin-lysozyme-EDTA-thallous acetate can be used to isolate $B$. anthracis from contaminated specimens, and bicarbonate agar is used as an identification method to induce capsule formation. Bacillus spp. usually grows within 24 hours of incubation at $35^{\circ} \mathrm{C}$, in ambient air (room temperature) or in $5 \% \mathrm{CO}_{2}$. If bicarbonate agar is used for identification, then the medium must be incubated in $5 \%$ $\mathrm{CO}_{2}$. B. anthracis colonies are medium-large, gray, flat, and irregular with swirling projections, often referred to as having a "medusa head" appearance, and are not hemolytic on 5\% sheep blood agar. The bacteria are not motile, susceptible to penicillin, and produce a wide zone of lecithinase on egg yolk agar. Confirmatory testing to identify $B$. anthracis includes gamma bacteriophage testing, indirect hemagglutination, and enzyme linked immunosorbent assay to detect antibodies [8]. The best confirmatory precipitation test for anthrax is the Ascoli test.

\subsection{Advanced Diagnostic Test}

PCR detection systems have been developed for B. anthracis (Beyer et al., 1996) and they become totally stand-alone and generally available for use in the non-specialist laboratory. A positive by this method is close to being accepted on a stand-alone basis now for clinical specimens and simple environmental samples such as tap water and air samples. In case the reason for a negative by PCR is the presence of inhibitors, negatives may need bacteriological support. Clinical specimens and more microbiologically "complex" samples, such as faeces, muddy water or soil, will generally need a DNA extraction stage and will also need bacteriological support to confirm positives or negatives. Rapid immunoassays for detection or confirmation of identity of $B$. anthracis are available commercially. The absolute specificities of these are, as yet, unproven and positives using these generally need to be confirmed bacteriologically or by PCR, or both [8]. The updated immunochromatographic device described by Muller et al. (2004) utilizes a monoclonal capture antibody to the anthrax protective antigen (PA) bound to a nitrocellulose membrane, and a second monoclonal antibody specific for a different epitope of PA bound to colloidal gold particles which become visible when they accumulate at the capture sites. The assay can detect as little as $25 \mathrm{ng} / \mathrm{ml}$ of PA and is performed in a few minutes without the need for special reagents. It therefore lends itself to direct diagnosis of cases of anthrax by detection of PA in the blood or body fluids, or to retrospective analysis of extracts from the types of sample of animal origin for which the Ascoli test was designed. As such, it has excellent specificity and sensitivity, but it has not become commercially available [3].

\section{Treatment and Prophylaxis}

\subsection{Treatment}

Livestock at risk should be immediately treated with a long-acting antibiotic to stop all potential incubating infections. Domestic livestock respond well to penicillin if treated in the early stages of the disease. Oxytetracycline given daily in divided doses also is effective. This is followed by vaccination $7-10$ days after antibiotic treatment. Antibiotics should not be administered within 1 wk of vaccination any animals becoming sick after initial treatment and/or vaccination should be retreated immediately and revaccinated a month later. Simultaneous use of antibiotics and vaccine is inappropriate, as available commercial vaccines for animals are live vaccines [6]. Animals should be moved to another pasture away from where the bodies had lain and any possible soil contamination. All cattle on neighboring premises should also be vaccinated. A buffer zone, 20-30 Km wide, is to be established around the infected area with in which all cattle and exposed sheep are vaccinated and quarantined.

\subsection{Vaccination}

The most widely used vaccine for prevention of anthrax in worldwide was developed by Max Sterne in 1937. He derived a rough variant of virulent $\mathrm{B}$. anthracis from culture on serum agar in an elevated $\mathrm{CO}_{2}$ atmosphere. This variant, named 34F2 sterne strain, was incapable of forming a poly-glutamic acid capsule and was subsequently found to have lost the pX02 plasmid, which codes for capsule formation. The immunity is established in ten days following inoculation. The animals are protected against natural infection for about one year.The main advantages of this strain are it is stable, safe in all animals, induces solid immunity lasting for one year, the immunogenicity can be tested in laboratory animals and has been used safely worldwide by laboratory staff involved in its preparation and by hundreds of thousands of veterinarians [9]. 


\section{Steps to be Undertaken to Prevent Anthrax Epidemics}

\subsection{Effective Carcass Disposal}

Avoid performing an autopsy when anthrax is being suspected. Ensure that all body openings anus, mouth, ears and nose are plugged with an absorbent material like non-perforated paper towel and cloths to prevent leakage of exudates. To prevent scavenging and spreading of spores by insects, birds, or mammals, once all body openings are plugged and the head securely covered, cover the carcass with a tarp, heavy plastic, or other appropriate material. Weigh down the edges of the covering to prevent removal by wind or predators. Under specific environmental conditions for instance, prolonged rain; carcass inaccessibility (e.g. standing water, heavy bush); or logistical problems (e.g. lack of proper equipment, manpower etc.) the prompt disposal of infected carcasses may be impossible. In these circumstances, to prevent or minimize anthrax environmental contamination, assess the situation to decide on an appropriate course of action within a realistic timeline for disposal. Cover the carcass and the surrounding area with disinfectants, such as $10 \%$ formalin or $5 \%$ solution of sodium hydroxide [10].

The goal is to destroy as many spores as possible, thereby decreasing environmental anthrax contamination. A complete burn should be achieved. The carcass should be completely reduced to ash. An effective burn primarily leaves ash and bits of bone, with minimal fly attraction to the site. If incineration is not feasible or cannot take place immediately, deep burial may be a viable option. The location of burial sites, using GPS or other mapping methods, should be recorded by those involved, and kept indefinitely. Be aware that burial permits may be required by municipal or provincial governing authorities and it should be away from water bodies. Ensure that the pit is 6-8 feet $(2 \mathrm{~m})$ deep - the bottom of which should be well above the water table (minimum 3 feet $(0.9 \mathrm{~m})$. Clay soil is preferable, whereas, sand or gravel should be avoided. Have a minimum of 3.2 feet $(1 \mathrm{~m})$ of clay at the base of the pit, and cover the carcass with a minimum of 3.2 feet $(1 \mathrm{~m})$ of clay and topsoil to prevent access by scavengers. Use 10\% formalin or $5 \%$ lye solution (sodium hydroxide) to decontaminate the carcass and the soil [10].

\subsection{Effect of Lime or Other Calcium Products on Spore Survival}

There is strong evidence that during the process of spore formation, calcium may be taken up from the sporulation environment and incorporated into the various layers of the spore. Most of the calcium, approximately $95 \%$, is located in the core region. Here, calcium, in combination with dipicolinic acid, forms a salt lattice that stabilizes the DNA and enzymes in the core, which is essential in the maintenance of dormancy and the thermoresistance properties of the spore. Approximately $5 \%$ of the calcium content of a spore is incorporated into the spore integument, where it appears to play an important role in the resistance, viability and future germinative capabilities of the spore. This evidence suggests that calcium increases the resilience of Bacillus spp. spores; therefore, the use of lime (calcium oxide) in anthrax disinfection, rather than destroying the spores, may actually aid in their long-term preservation. As well, lime has the added disadvantage of providing spores with the basic (high $\mathrm{pH}$ ) environment that is optimal for calcium enrichment [4]. The addition of lime to a putrefying, and thus heat producing, carcass may be especially problematic, since high temperature is another catalyst for calcium adsorption. This concludes that we should not to use the lime or any other calcium containing compound on carcasses and contaminated ground in the anthrax outbreaks [2].

Peracetic acid, a peroxide-based disinfectant has a promising effect on anthrax disinfection both because of its sporucidal properties and because it has the additional benefit of creating an acidic environment that may facilitate calcium leaching from spores and loss of spore viability and 5\% solution of formaldehyde will also has sporucidal effect .

\subsection{Handling the Premises}

The site where the animal died should be disinfected with 5\% formaldehyde or peracetic acid to minimize anthrax environmental contamination. Affected premises are to be quarantined for at least 20 days after disposal of the carcass and disinfection [10].

\section{Conclusion}

The repeated anthrax outbreak in livestock and subsequent infection to human has been considered as a nationwide alarming issue. This review focuses on certain ecological factors that might be responsible for survival of anthrax spore in soil, e.g. soil type, calcium content, organic carbon content and soil $\mathrm{pH}$. Effective carcass disposal and disinfection of site prevents contaminating the ground with the anthrax spores or organisms. After an outbreak, quarantine the healthy livestock, vaccinate and move them away to clean pastures reduces anthrax epidemics.

\section{References}

[1] J.T. Bartlett, L. Inglesby and Borio. Management of anthrax. Clin. Infect. Dis. 35: 851-857. 2002.

[2] F.W. Eurich and M.D. Edin. The history of anthrax in the wool industry of Bradford, and of its control. Lancet. January 2, 57-58, 1926.

[3] D. Dragon, and R. Rennie. The ecology of anthrax spores: tough but not invincible. Can Vet J. 36:295-301. 1995.

[4] K.Ibrahim, G. Brown, D. Wright and J. Rotschafer. Bacillus anthracis: medical issues of biologic warfare. Pharmacotherapy. 19: 690-701.1990.

[5] S.E. Pabian and M.C. Brittingham. Soil calcium and forest birds: Indirect links between nutrient availability and community composition.Ecosystems 15: 748-760. 2012. 
[6] S. Leppl. Anthrax toxins. In J. Moss, B. Iglewski, M. Vaughan, and $\mathrm{A}$. T. Tu (ed.) Bacterial toxins and virulence factors in disease. p. 543-572.1995.

[7] M.N.Van Ert, W.R. Easterday, L.Y.Huynh, R.T.Okinaka, M.E. Hugh-Jones J Ravel, Global genetic population structure of Bacillus anthracis. PLOS ONE. 2:461 2007.

[8] P.C.B Turnbull. Definitive identification of Bacillus anthracis - a review. J. Appl. Microbiol.87:237-240. 1999.
[9] M. Sterne. The effect of different carbon dioxide concentrations on the growth of virulent anthrax strains. Onderstepoort J. Vet. Sci. Anim. Ind., 9, 49-67.1937.

[10] M.N. Mongoh, N.W. Dyer, C.L. Stoltenow and M.L Khaitsa. Risk factors associated with anthrax outbreak in animals in North Dakota, 2005: A retrospective case-control study. Public Health Rep 123: 352-359. 2008. 